\title{
INDUCTIVE DEFINITIONS AND COMPUTABILITY $\left({ }^{1}\right)$
}

\author{
BY \\ THOMAS J. GRILLIOT
}

\begin{abstract}
Sets inductively defined with respect to $\Pi_{0}, \Sigma_{1}$, (nonmonotonic) $\Pi_{1}$ and $\Sigma_{2}$ predicates are characterized in terms of the four chief notions of abstract recursion.
\end{abstract}

Moschovakis [7] has introduced the notions of prime and search computability on an abstract set. Both notions of computability are generalizations of general recursiveness since a function on the natural numbers is general recursive iff it is search [alternatively, prime] computable in the successor function (and 0 , if constant functions are not automatically considered computable). For structures that have no "natural" search operator, search computability is generally a stronger notion than prime computability. Analogously, prime and search computability in $\boldsymbol{E}$, the type 2 object that embodies quantification over the given domain, are generalizations of hyperarithmeticalness. Moschovakis [8] and Gordon [3] have shown that other abstract notions of recursion such as those of Fraïssé [2], Lacombe [5] and Montague [6] are equivalent to search computability. Moschovakis [8], [9] and Barwise-Gandy-Moschovakis [1] have shown that some abstract notions of hyperarithmeticalness are equivalent to search computability in $\boldsymbol{E}$. In this paper we will characterize notions of abstract computability in terms of various inductively defined sets. Let PC and SC be abbreviations for prime computable and search computable. Let "built up by" be an abbreviation for "reducible to a set inductively defined with respect to". Our main result is to show the following equivalences for predicates:

(1) Semi-PC $\Leftrightarrow$ built up by $\Pi_{0}$ predicate (Theorems 2 and 6).

(2) Semi-SC $\Leftrightarrow$ built up by monotonic $\Sigma_{1}$ predicate (Theorems 1 and 5).

(3) Semi-(PC in $E) \Leftrightarrow$ built by $\Pi_{1}$ predicate (Theorems 4 and 8 ).

(4) Semi-(SC in $E) \Leftrightarrow$ built up by $\Sigma_{2}$ predicate (Theorems 3 and 7).

These results relativize to a list of relations, and "monotonic" may be inserted before $\Pi_{0}$ and $\Sigma_{2}$ in equivalences (1) and (4). However, "monotonic" may not usually be inserted before $\Pi_{1}$ in equivalence (3) and "monotonic" may never be deleted from equivalence (2). After we have introduced the necessary definitions, we shall point out why equivalences (2) and (3) are so sensitive to monotonicity.

Received by the editors June 30, 1969.

AMS 1970 subject classifications. Primary 02F27; Secondary 02F35.

Key words and phrases. Inductively defined sets, abstract computability, hyperprojective hierarchy.

( ${ }^{1}$ Some of the results contained in this paper can be found, in a slightly different context, in the author's dissertation written under the direction of J. R. Shoenfield. 
The reader should be familiar with at least the first few pages of Moschovakis [7] because the schemes for prime computability (in $\boldsymbol{E}$ ) and search computability (in $\boldsymbol{E})$ are given there. If a predicate $P(\mathfrak{a})$ can be expressed in the form $\{f\}(\mathfrak{a}) \downarrow$, then it is called semi-PC or semi-SC according as the index $f$ is a PC index or a SC index. If the use of scheme $\mathrm{Cl}$ (scheme that introduces constants) is restricted to introducing only those constants formed from 0 and the pairing operation $($,$) , then$ the word absolutely is prefixed before PC and SC.

Greek letters will be used for ordinals, and lower case italic letters for elements of $B^{*}$, where $B^{*}$ is described in [7, p. 428]. $S$ and $T$ will be used for subsets of $B^{*}$. Following Spector [11], the $\sigma$ th stage $S_{\sigma}$ of a set $S$ inductively defined with respect to a predicate $Q$ is given transfinitely by the rule

$$
x \in S_{\sigma} \Leftrightarrow \exists \tau<\sigma, Q\left(x, S_{\tau}\right),
$$

and $S$ is the union of the $S_{\sigma}$. Sometimes equivalence (5) will be expressed more informally thus: $x \in S$ if $Q(x, S)$. Each $x \in S$ can be assigned an ordinal $|x|$ equal to the first ordinal $\sigma$ satisfying $x \in S_{\sigma+1}$. If $Q$ satisfies the property

$$
Q\left(x, T_{1}\right) \& T_{1} \subseteq T_{2} \Rightarrow Q\left(x, T_{2}\right),
$$

for all $x, T_{1}, T_{2}$, then $Q$ is called monotonic.

A predicate $Q(x, T)$ is $\Pi_{n}$ [resp., $\Sigma_{n}$ ] if its quantifiers in prenex form begin with universal [resp., existential] and alternate in kind at most $n-1$ times and if its matrix is formed from the usual connectives $(\&, \vee, \neg)$, equality $(=)$, zero $(0)$, pairing $(()$,$) , projections (\pi, \delta)$, variables ranging through elements of $B^{*}$ and the symbols $\in$ and $T$ which must occur together thus: $\ldots \in T$. For example, $\exists x \forall y\left((x)_{0} \in T \vee y=((x, z), 0)\right)$ is $\Sigma_{2}$. If additional relations $R_{1}, \ldots, R_{n}$ are used in the matrix, we write $\Pi_{n}\left(R_{1}, \ldots, R_{n}\right)$ [resp., $\Sigma_{n}\left(R_{1}, \ldots, R_{n}\right)$ ] to emphasize this. An important class of monotonic $\Pi_{n}$ [resp., $\Sigma_{n}$ ] predicates are the positive ones. These predicates have the property that each occurrence of $\ldots \in T$ is in the scope of an even number of $\neg$ 's. For all the theorems of this paper, the words "positive" and "monotonic" are interchangeable. A predicate $P(\mathfrak{a})$ is reducible to a set $T$ if, for all $a$,

$$
P(\mathfrak{a}) \Leftrightarrow\langle 0, \mathfrak{a}\rangle \in T .
$$

(This is more restrictive than the usual definition of reducibility, but it is strong enough for our purposes.)

Let us illustrate why equivalences (2) and (3) are sensitive to monotonicity. Let $U$ be some $\Pi_{1}$ set, say, for simplicity, $x \in U \Leftrightarrow \neg \exists y\left(\left\langle x,(y)_{0}\right\rangle=y\right)$. Let $Q(x, T)$ be

$$
\begin{aligned}
& x=\langle 0\rangle \quad \vee \\
& x=\left\langle 1,(x)_{1}\right\rangle \& \exists y\left(\left\langle(x)_{1},(y)_{0}\right\rangle=y\right) \\
& x=\left\langle 2,(x)_{1}\right\rangle \&\langle 0\rangle \in T \&\left\langle 1,(x)_{1}\right\rangle \notin T .
\end{aligned}
$$

Clearly the first three stages of the set inductively defined with respect to $Q$ are $S_{0}=\varnothing, S_{1}=\{\langle 0\rangle\} \cup\{\langle 1, x\rangle: x \notin U\}$ and $S_{2}=S_{1} \cup\{\langle 2, x\rangle: x \in U\}$. Thus $U$ is 
built up by a $\Sigma_{1}$ predicate $Q$ by first building $U^{c}$ and then "complementing." By "complementing" several times, $\Sigma_{1}$ predicates can build up all $\Sigma_{n}$ sets and, in fact, all $\bigcup_{n} \Sigma_{n}$ sets where $\bigcup_{n}$ denotes "recursive" union. Conversely, by an application of the first half of Theorem 5 , any set inductively defined with respect to a $\Sigma_{1}$ predicate is a $\bigcup_{n} \Sigma_{n}$ set. Since there are non-SC $\bigcup_{n} \Sigma_{n}$ sets, this illustrates that nonmonotonic $\Sigma_{1}$ predicates can build more complex sets than monotonic $\Sigma_{1}$ predicates can. Monotonicity prevents the use of "complementing." A similar observation holds when $\Sigma_{1}$ is replaced by $\Pi_{1}$. This explains why equivalences (2) and (3) cannot be altered by deleting or inserting the word "monotonic." A related comment regarding equivalence (4) is in order. If the inductive defining predicates are assumed to be positive, their forms can be more complex than $\Sigma_{2}$, e.g., $\Sigma_{n}$ or $\Pi_{n}$ or even semi-(SC in $\left.\boldsymbol{E}\right)$ as shown by Moschovakis [7]. However, if no monotonicity condition is assumed, $\Sigma_{2}$ predicates are best possible in that $\Pi_{2}$ predicates can build up sets more complex than semi-(SC in $\boldsymbol{E})$ sets. No one has satisfactorily classified the complexity of general $\Pi_{2}$ inductive definitions even in the case for natural numbers, though Richter [10] has made some contribution. (Added in proof: Recently Aczel and Richter solved this problem for natural numbers.)

THEOREM 1. For $S C$ in $=, R_{1}, \ldots, R_{n}$, the predicates $\{f\}(\mathfrak{a}) \downarrow$ and $\{f\}(\mathfrak{a}) \rightarrow z$ are reducible to a set inductively defined with respect to a monotonic $\Sigma_{1}\left(R_{1}, \ldots, R_{n}\right)$ predicate.

Proof. We drop $R_{1}, \ldots, R_{n}$ for simplicity. The idea is to inductively define a set $S$ so that

$$
\{f\}^{=}(\mathfrak{a}) \downarrow \Leftrightarrow\langle 0, f, \mathfrak{a}\rangle \in S, \quad\{f\}=(\mathfrak{a}) \rightarrow z \Leftrightarrow\langle 1, f, z, \mathfrak{a}\rangle \in S .
$$

For each of the schemes $\mathrm{C} 0$ to $\mathrm{C} 9$, we merely set up two clauses for inductively defining $S$. Let us consider the two examples corresponding to C2 and C5. Let $n$ denote the length of the list $\mathfrak{a}$. Since $y=\{\langle 2, n+1\rangle\}=(y, \mathfrak{a})$, we let

$$
\langle 0,\langle 2, n+1\rangle, y, \mathfrak{a}\rangle \in S, \quad\langle 1,\langle 2, n+1\rangle, y, y, \mathfrak{a}\rangle \in S,
$$

and since $\{\langle 5, n, g, h\rangle\}=(\mathfrak{a}) \cong\{g\}=(\{h\}=(\mathfrak{a}), \mathfrak{a})$, we let

$$
\begin{aligned}
\langle 0,\langle 5, n, g, h\rangle, \mathfrak{a}\rangle \in S & \text { if } \exists z[\langle 0, g, z, \mathfrak{a}\rangle \in S \&\langle 1, h, z, \mathfrak{a}\rangle \in S], \\
\langle 1,\langle 5, n, g, h\rangle, y, \mathfrak{a}\rangle \in S & \text { if } \exists z[\langle 1, g, y, z, \mathfrak{a}\rangle \in S \&\langle 1, h, z, \mathfrak{a}\rangle \in S] .
\end{aligned}
$$

Though these clauses are not quite in the form $x \in S$ if $Q(x, S)$, but rather in the form $\langle\cdots\rangle \in S$ if $Q(\langle\cdots\rangle, S)$, we can remedy this by inductively defining as part of $S$ the set $\{\langle 2, x\rangle:$ Seq $(x)\}$. Then for example the clauses in (6) may be rewritten thus:

$$
\begin{array}{ll}
x \in S & \text { if }\langle 2, x\rangle \in S \&(x)_{0}=0 \&(x)_{1}=\left\langle 2,\left((x)_{1}\right)_{1}\right\rangle \&\left((x)_{1}\right)_{1}+2=\pi x, \\
x \in S & \text { if }\langle 2, x\rangle \in S \&(x)_{0}=1 \&(x)_{1}=\left\langle 2,\left((x)_{1}\right)_{1}\right\rangle \&\left((x)_{1}\right)_{1}+2=\pi x \&(x)_{2}=(x)_{3} .
\end{array}
$$


Thus we obtain an inductive definition of $S$ with respect to a $\Sigma_{1}$ predicate (existential quantifiers are used for the clauses corresponding to $\mathrm{C5}, \mathrm{C6}$ and $\mathrm{C} 9$ ). Schemes C7 and C8 pose the slight problems of permuting a list of variables of nonfixed length and of dropping a list of variables of nonfixed length. These problems are handled by adding some auxiliary clauses to the inductive definition of $S$.

THEOREM 2. For $P C$ in $=, R_{1}, \ldots, R_{n}$, the predicates $\{f\}(\mathfrak{a}) \downarrow$ and $\{f\}(\mathfrak{a}) \rightarrow z$ are reducible to a set inductively defined with respect to a monotonic $\Pi_{0}\left(R_{1}, \ldots, R_{n}\right)$ predicate.

Proof. The set $S$ in the proof of Theorem 1 has existential quantifiers in its inductive definition in only the clauses corresponding to C5, C6 and C9. For this theorem we drop the clauses corresponding to C9. The remaining existential quantifiers may be removed in accordance with the following two observations. First, each existential quantifier $\exists z$ occurring in the clauses for $\mathrm{C} 5$ and $\mathrm{C} 6$ may be replaced by $\exists z \in[\mathfrak{b}]$ where $\mathfrak{b}$ is the list of free variables occurring in the scope of the quantifier. ([ $[\mathfrak{b}]$ is the set of all objects formed from $0, \mathfrak{b}$ and the pairing and projection functions.) For instance, the first clause in (7) may be altered by inserting " $\in[g, h, \mathfrak{a}]$ " after the quantifier $\exists z$. This follows from Lemma 24 of [7] that shows that the value of $\{f\}(\mathfrak{a})$ is in $[f, \mathfrak{a}]$ when $f$ is a $\mathrm{PC}$ index. The second observation is that quantifiers of the form $\exists z \in[\mathfrak{b}]$ can be built up by a monotonic $\Pi_{0}$ predicate, as is exemplified in the following lemma. Thus with slight alterations the set $S$ described in the preceding theorem (excluding clauses for C9) can be inductively defined with respect to a monotonic $\Pi_{0}$ predicate.

LEMMA. If $S$ is inductively defined with respect to some predicate, then a few $\Pi_{0}$ clauses can be added to its inductive definition so that the new inductively defined set $T$ satisfies the property

$$
\exists z \in[\mathfrak{b}](\langle 0, z, \mathfrak{b}\rangle \in T) \Rightarrow\langle 1, \mathfrak{b}\rangle \in T .
$$

Thus quantifiers of the form $\exists z \in[\mathfrak{b}]$ can be "built up" by $\Pi_{0}$ predicates.

Proof. For simplicity consider the case where $\mathfrak{b}$ is $x, y$, and $x, y \in B$. Add to the inductive definition of $S$ the following clauses:

$$
\begin{aligned}
\langle 2, x, y, z\rangle \in T & \text { if }\langle 0, z, x, y\rangle \in T, \\
\langle 2, x, y, u, v, \mathfrak{a}\rangle \in T & \text { if }\langle 2, x, y,(u, v), \mathfrak{a}\rangle \in T, \\
\langle 2, x, y, \mathfrak{a}\rangle \in T & \text { if }\langle 2, x, y, x, \mathfrak{a}\rangle \in T \text { or }\langle 2, x, y, y, \mathfrak{a}\rangle \in T \text { or }\langle 2, x, y, 0, \mathfrak{a}\rangle \in T, \\
\langle 1, x, y\rangle \in T & \text { if }\langle 2, x, y\rangle \in T .
\end{aligned}
$$

For example, suppose $\exists z \in[x, y](\langle 0, z, x, y\rangle \in T)$; say $\langle 0,((x, y), 0), x, y\rangle \in T$. Then the following objects are successively put into $T$ :

$$
\begin{gathered}
\langle 2, x, y,((x, y), 0)\rangle,\langle 2, x, y,(x, y), 0\rangle,\langle 2, x, y, x, y, 0\rangle, \\
\langle 2, x, y, y, 0\rangle,\langle 2, x, y, 0\rangle,\langle 2, x, y\rangle,\langle 1, x, y\rangle .
\end{gathered}
$$

Thus $\langle 1, x, y\rangle \in T$, which is what we want. 
Theorem 3. For $S C$ in $E,=, R_{1}, \ldots, R_{n}$, the predicates $\{f\}(\mathfrak{a}) \downarrow$ and $\{f\}(\mathfrak{a}) \rightarrow z$ are reducible to a set inductively defined with respect to a monotonic $\Pi_{1}\left(R_{1}, \ldots, R_{n}\right) \vee$ $\Sigma_{1}\left(R_{1}, \ldots, R_{n}\right)$ predicate.

(This result occurs in Moschovakis [7, p. 493].)

Proof. Modify the inductive definition of $S$ described in Theorem 1 by adding the following clauses for scheme $\mathrm{C10}$. It is desirable to maintain the property that, whenever $\{f\}(\mathfrak{a}) \rightarrow z,|\langle 0, f, \mathfrak{a}\rangle|=|\langle 1, f, z, \mathfrak{a}\rangle|$ (i.e., both objects are put into $S$ at the same stage); so the first clause is inserted to maintain this synchronization.

$$
\begin{aligned}
\langle 7,\langle 10, n, g\rangle, \mathfrak{a}\rangle \in S & \text { if } \forall y(\langle 0, g, y, \mathfrak{a}\rangle \in S), \\
\langle 0,\langle 10, n, g\rangle, \mathfrak{a}\rangle \in S & \text { if }\langle 7,\langle 10, n, g\rangle, \mathfrak{a}\rangle \in S, \\
\langle 8, g, y, \mathfrak{a}\rangle \in S & \text { if } \exists u(u \neq 0 \&\langle 1, g, u, y, \mathfrak{a}\rangle \in S), \\
\langle 1,\langle 10, n, g\rangle, 1, \mathfrak{a}\rangle \in S & \text { if }\langle 7,\langle 10, n, g\rangle, \mathfrak{a}\rangle \in S \& \forall y(\langle 8, g, y, \mathfrak{a}\rangle \in S), \\
\langle 1,\langle 10, n, g\rangle, 0, \mathfrak{a}\rangle \in S & \text { if }\langle 7,\langle 10, n, g\rangle, \mathfrak{a}\rangle \in S \& \exists y(\langle 1, g, 0, y, \mathfrak{a}\rangle \in S) .
\end{aligned}
$$

Theorem 4. For $P C$ in $E,=, R_{1}, \ldots, R_{n}$, the predicates $\{f\}(\mathfrak{a}) \downarrow$ and $\{f\}(\mathfrak{a}) \rightarrow z$ are reducible to a set inductively defined with respect to $a \Pi_{1}\left(R_{1}, \ldots, R_{n}\right)$ predicate.

Proof. Modify the inductive definition of $S$ described in Theorem 2 by adding the following clauses for scheme $\mathrm{C} 10$. Note that these clauses are similar to those used in the preceding proof except that existential quantifiers have been avoided by using universal quantifiers at the expense of monotonicity.

$$
\begin{aligned}
\langle 7,\langle 10, n, g\rangle, \mathfrak{a}\rangle \in S & \text { if } \forall y(\langle 0, g, y, \mathfrak{a}\rangle \in S), \\
\langle 0,\langle 10, n, g\rangle, \mathfrak{a}\rangle \in S & \text { if }\langle 7,\langle 10, n, g\rangle, \mathfrak{a}\rangle \in S, \\
\langle 8, g, \mathfrak{a}\rangle \in S & \text { if } \forall y(\langle 0, g, y, \mathfrak{a}\rangle \in S) \& \forall y(\langle 1, g, 0, y, \mathfrak{a}\rangle \notin S), \\
\langle 1,\langle 10, n, g\rangle, 1, \mathfrak{a}\rangle \in S & \text { if }\langle 8, g, \mathfrak{a}\rangle \in S, \\
\langle 1,\langle 10, n, g\rangle, 0, \mathfrak{a}\rangle \in S & \text { if }\langle 7,\langle 10, n, g\rangle, \mathfrak{a}\rangle \in S \&\langle 8, g, \mathfrak{a}\rangle \notin S .
\end{aligned}
$$

For example, suppose $\{\langle 10, n, g\rangle\}^{E,}=(\mathfrak{a}) \rightarrow 0$; that is $\{g\}^{E,}=(y, \mathfrak{a}) \downarrow$ for all $y$ and $\{g\}^{E,=}(y, \mathfrak{a}) \rightarrow 0$ for some $y$. Let $\sigma$ be the first ordinal for which $\langle 0, g, y, \mathfrak{a}\rangle \in S_{\sigma}$ for all $y$. Since $\langle 1, g, 0, y, \mathfrak{a}\rangle \in S_{\sigma}$ for some $y$, we have $\langle 7,\langle 10, n, g\rangle, \mathfrak{a}\rangle \in S_{\sigma+1}$ but $\langle 8, g, \mathfrak{a}\rangle \notin S_{\sigma+1}$; so $\langle 0,\langle 10, n, g\rangle, \mathfrak{a}\rangle \in S_{\sigma+2}$ and $\langle 1,\langle 10, n, g\rangle, 0, \mathfrak{a}\rangle \in S_{\sigma+2}$, which is what we want.

Now we will prove the converses of Theorems 1 through 4 .

LeMma. If $P(\mathfrak{a}, T)$ is $\Pi_{0}$ and $T=\bigcup_{i<\omega} T_{i}$ where $T_{i} \subseteq T_{j}$ for $i<j, P(\mathfrak{a}, T) \Leftrightarrow P\left(\mathfrak{a}, T_{\mathfrak{i}}\right)$ for all sufficiently large $i$.

Proof. $T$ occurs in $P$ only finitely often, say, in $b_{1} \in T, \ldots, b_{k} \in T$. Let $i$ be large enough so that $\left\{b_{1}, \ldots, b_{k}\right\} \cap T \subseteq T_{i}$. Then $P(\mathfrak{a}, T)$ iff $P\left(\mathfrak{a}, T_{i}\right)$.

THEOREM 5. A set inductively defined with respect to a $\Sigma_{1}\left(R_{1}, \ldots, R_{n}\right)$ predicate has at most $\omega$ stages. $A$ set inductively defined with respect to a monotonic $\Sigma_{1}\left(R_{1}, \ldots, R_{n}\right)$ predicate is absolutely semi-(SC in $\left.=, R_{1}, \ldots, R_{n}\right)$. 
Proof. For simplicity, drop $R_{1}, \ldots, R_{n}$. Suppose $S$ is inductively defined with respect to $\exists y P(x, y, T)$ where $P$ is $\Pi_{0}$. If $P\left(x, y, S_{\omega}\right)$ is true for some $y$, then, by the lemma, $P\left(x, y, S_{n}\right)$ is true for some $n \in \omega$. It follows that $S_{\omega+1}=S_{\omega}=S$.

Next suppose that $\exists y P(x, y, T)$ is monotonic.

$$
\begin{aligned}
x \in S \leftrightarrow & \exists n \in \omega\left[x \in S_{n}\right] \\
\leftrightarrow & \exists n \in \omega \exists \text { finite sets } T_{0}, \ldots, T_{n} \\
& {\left[x \in T_{n} \& \forall i<n T_{i+1} \subseteq\left\{z: \exists y P\left(z, y, T_{i}\right)\right\}\right] } \\
\leftrightarrow & \exists n \in \omega \exists z_{0}, z_{1}, \ldots, z_{n} \\
& {\left[x=\left(z_{n}\right)_{0} \& \forall i<n \forall j<\ln \left(z_{i+1}\right) \exists y P\left(\left(z_{i+1}\right)_{j}, y,\left\{\left((z)_{i}\right)_{k}: k<\operatorname{lh}\left(z_{i}\right)\right\}\right)\right] . }
\end{aligned}
$$

Thus the predicate $x \in S$ may be put in the form $\exists y[\{f\}=(x, y) \rightarrow 0]$ for some absolute index $f$, which in turn may be put in the form $\{\langle 9,2, f\rangle\}=(x) \downarrow$.

THEOREM 6. A set inductively defined with respect to a $\Pi_{0}\left(R_{1}, \ldots, R_{n}\right)$ predicate is absolutely semi- $\left(P C\right.$ in $\left.=, R_{1}, \ldots, R_{n}\right)$.

Proof. For simplicity drop $R_{1}, \ldots, R_{n}$. Suppose $S$ is inductively defined with respect to $P(x, T)$ where $P$ is $\Pi_{0}$. By the recursion theorem, there exists a function $F$ that is absolutely $\mathrm{PC}$ in $=$ satisfying

$$
\begin{aligned}
F(x, y)=0 & \text { if } y \neq 0 \& P\left(x,\left\{z: F\left(z,(y)_{0}\right)=0\right\}\right), \\
=0 & \text { if } F\left(x,(y)_{0}\right)=0, \\
=1 & \text { otherwise. }
\end{aligned}
$$

Then, for each $n \in \omega, F(x, n)=0 \Leftrightarrow x \in S_{n}$; so $x \in S \Leftrightarrow \mu n(F(x, n)=0) \downarrow$.

Definitions. Before we prove Theorems 7 and 8 , we need some notation. To each defined $\{f\}^{E,=}(\mathfrak{a})$, an ordinal $\left|\{f\}^{E,=}(\mathfrak{a})\right|$ can be assigned whose size indicates the size of the tree of computation of $\{f\}^{E,=}(\mathfrak{a})$. A precise definition is given by Moschovakis [7, pp. 444-445]. Call ordinals $\sigma_{\mathfrak{a}} \mathrm{SC}$ [resp., PC] in $\boldsymbol{E},=, \mathfrak{a}, \mathfrak{b}$ (uniformly in $\mathfrak{a}$ ) if, for some absolute SC [resp., PC] index $f, \sigma_{\mathfrak{a}}=\left|\{f\}^{E=}=(\mathfrak{a}, \mathfrak{b})\right|$ where $\{f\}^{E,=}(\mathfrak{a}, \mathfrak{b}) \downarrow$. Note that, if $\sigma_{z}$ is SC [resp., PC] in $\boldsymbol{E},=, z, \mathfrak{a}$ (uniformly in $z$ ), then there exists an ordinal $\tau \geqq \sup _{z} \sigma_{z}$ that is SC [resp., PC] in $E$, $=$, a. (Reason: $\left|\{g\}^{E,=}(\mathfrak{a})\right| \geqq \sup _{z}\left|\{f\}^{E,=}(z, \mathfrak{a})\right|$ where $\{g\}^{E,=}(\mathfrak{a}) \cong E\left(\lambda_{z}\{f\}^{E,=}(z, \mathfrak{a})\right)$.) Let $\kappa^{R_{1}, \ldots, R_{n}}$ [resp., $\left.\lambda^{R_{1}}, \ldots, R_{n}\right]$ be the supremum of all ordinals $\mathrm{SC}$ [resp., PC] in $E,=, R_{1}, \ldots, R_{n}, x$ for some $x$.

THEOREM 7. A set inductively defined with respect to a $\Sigma_{2}\left(R_{1}, \ldots, R_{n}\right)$ predicate is absolutely semi-( $S C$ in $\left.E,=, R_{1}, \ldots, R_{n}\right)$ and has at most $\kappa^{R_{1}}, \ldots, R_{n}$ stages.

Proof. For simplicity drop $R_{1}, \ldots, R_{n}$. Suppose $S$ is inductively defined with respect to $\exists y \forall z P(x, y, z, T)$, where $P$ is $\Pi_{0}$. We want to show that $S=S_{\kappa}$. If this is the case, then

$$
\begin{aligned}
x \in S & \Leftrightarrow \exists f \exists y\left[x \in S_{\sigma} \text { where } \sigma=\left|\{f\}^{E,}=(y)\right|\right] \\
& \Leftrightarrow \nu y\left[x \in S_{\sigma} \text { where } \sigma=\left|\left\{(y)_{0}\right\}^{E,=}\left((y)_{1}\right)\right|\right] \downarrow .
\end{aligned}
$$


Thus $S$ is absolutely semi-(SC in $E,=)$ since the predicate $\left[x \in S_{\sigma}\right.$ where $\left.\sigma=\left|\{f\}^{E}=(y)\right|\right]$ is absolutely $\mathrm{SC}$ in $E,=$. To see that $S_{\kappa+1}=S_{\kappa}$, suppose $\forall z P\left(x, a, z, S_{\kappa}\right)$ for some $a$. We will show that $\forall z P\left(x, a, z, S_{\alpha}\right)$ for some $\alpha<\kappa$; hence, if $x \in S_{x+1}$ then $x \in S_{x}$. In fact, letting $\alpha=\sup _{n \in \omega} \alpha_{n}$ where the $\alpha_{n}$ satisfy

$$
\alpha_{n+1} \geqq \sup _{z} \mu \beta\left[\beta \geqq \alpha_{n} \& P\left(x, a, z, S_{\beta}\right)\right] \text {, }
$$

we get $\forall z P\left(x, a, z, S_{\alpha}\right)$. To see this, let $z$ be arbitrary. There exist ordinals $\beta_{n}$ such that $\alpha_{n} \leqq \beta_{n} \leqq \alpha_{n+1}$ and $P\left(x, a, z, S_{\beta_{n}}\right)$ is true. Since $S_{\alpha}=\bigcup_{n} S_{\beta_{n}}$, by the lemma, $P\left(x, a, z, S_{\alpha}\right)$ is true. We are finished if we can make the $\alpha_{n}$ small enough so that $\alpha<\kappa$. We obtain the $\alpha_{n}$ by the following recursion. Suppose that $\alpha_{n}$ is SC in $\boldsymbol{E},=, n, x, a$ (uniformly in $n$ ). Since any $\beta$ close to $\kappa$ satisfies $\beta \geqq \alpha_{n} \& P\left(x, a, z, S_{\beta}\right)$, using the $\nu$-operator, we get one that is $\mathrm{SC}$ in $E,=, n, z, x, a$ (uniformly in $n, z$ ). Call it $\gamma_{n z}$. Using the recursion theorem construct $\alpha_{n+1} \geqq \sup _{z} \gamma_{n z}$. Since $\alpha$ $=\sup _{n \in \omega} \alpha_{n}$ has ordinals above it SC in $\boldsymbol{E},=, x, a$, we see that $\alpha<\kappa$.

Though we have no $\nu$-operator for PC functions, it is useful to have some sort of selection operator. We restrict our attention to $\mathrm{PC}$ functions. The predicate (with arguments $f, \mathfrak{a}, g, \mathfrak{b}$ )

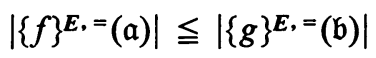

is absolutely $\mathrm{PC}$ in $\boldsymbol{E},=$ and is defined whenever one of $\{f\}^{E,=}(\mathfrak{a})$ and $\{\boldsymbol{g}\}^{E,=}(\mathfrak{b})$ is defined. One proves this by the recursion theorem: to compare $\left|\{f\}^{E,=}(\mathfrak{a})\right|$ and $\left|\{g\}^{E,=}(b)\right|$, one computes appropriate comparisons between ordinals of the form $\left|\{h\}^{E,=}(\mathfrak{c})\right|$ and $\left|\{i\}^{E,=}(\mathfrak{D})\right|$ where $\{h\}^{E,=}(\mathfrak{c})$ and $\{i\}^{E,=}(\mathfrak{D})$ are respectively in the trees of computation of $\{f\}^{E,}=(\mathfrak{a})$ and $\{g\}^{E,=}(\mathfrak{D})$; hence a recursion. Another way of solving the problem is to show that, for the set $S$ inductively defined in the proof of Theorem 4, the predicate $|x| \leqq|y|$ is absolutely $\mathrm{PC}$ in $\boldsymbol{E},=$ and is defined whenever $x \in S$ or $y \in S$. This is shown as follows. There is a PC function $f(x, z, i)$ and a fixed number $k$ such that, if $x \in S$, then $|x|=0$ or $|x|=\sup _{z} \inf _{i \leqq k}|f(x, z, i)|$. This is easily seen by examining each of the clauses in the inductive definition of $S$. Then the methods in Theorems 1 and 2 of [4] can be applied to obtain a selection operator.

If $\{f\}^{E,=}(n, \mathfrak{a}) \downarrow$ for some $n \in \omega$, then it is useful to find such an $n$. Define $g$ by the recursion

Then

$$
\begin{aligned}
\{g\}^{E,=}(n, \mathfrak{a}) & \cong n & & \text { if }\left|\{f\}^{E,=}(n, \mathfrak{a})\right| \leqq\left|\{g\}^{E,=}(n+1, \mathfrak{a})\right|, \\
& \cong\{g\}^{E,=}(n+1, \mathfrak{a}) & & \text { if not. }
\end{aligned}
$$

$$
\exists n \in \omega\left[\{f\}^{E,=}(n, \mathfrak{a}) \downarrow\right] \Rightarrow\{f\}^{E,=}\left(\{g\}^{E,=}(0, \mathfrak{a}), \mathfrak{a}\right) \downarrow .
$$

Since absolute indices can be effectively enumerated, it follows that, given a predicate $P(f, \mathfrak{a}) \mathrm{PC}$ in $\boldsymbol{E},=$ there exists $h$ such that

$$
\begin{aligned}
\exists f \in\{\text { absolute } P C \text { indices }\}\left[\{f\}^{E,}=\right. & (\mathfrak{a}) \downarrow \& P(f, \mathfrak{a})] \\
& \Rightarrow\left\{\{h\}^{E,=}(\mathfrak{a})\right\}^{E,=}(\mathfrak{a}) \downarrow \& P\left(\{h\}^{E,=}(\mathfrak{a}), \mathfrak{a}\right) .
\end{aligned}
$$

This fact will be used twice in the next theorem. 
THEOREM 8. A set inductively defined with respect to a $\Pi_{1}\left(R_{1}, \ldots, R_{n}\right)$ predicate is absolutely semi-( $P C$ in $\left.E,=, R_{1}, \ldots, R_{n}\right)$ and has at most $\lambda^{R_{1}}, \ldots, R_{n}$ stages.

Proof. For simplicity drop $R_{1}, \ldots, R_{n}$. Suppose $S$ is inductively defined with respect to $\forall z P(x, z, T)$ where $P$ is $\Pi_{0}$. Let $\pi(\mathfrak{a})$ be an abbreviation for the supremum of all ordinals $\mathrm{PC}$ in $\boldsymbol{E},=$, a. We want to show that $x \in S \Rightarrow x \in S_{\pi(x)}$, in which case we see that $S=\bigcup_{x} S_{\pi(x)}=S_{\lambda}$ and

$$
\begin{aligned}
x \in S & \Leftrightarrow \exists f\left[f \text { is an absolute PC index \& } x \in S_{\sigma} \text { where } \sigma=\left|\{f\}^{E,}=(x)\right|\right] \\
& \Leftrightarrow\{g\}^{E,=}(x) \downarrow
\end{aligned}
$$

for some absolute PC index $g$. (The existence of $g$ is insured by the selectionoperator theorem.)

Let $x \in S$; so $\forall z P\left(x, z, S_{|x|}\right)$. We will show that $x \in S_{\pi(x)}$. As an induction hypothesis, we may assume that, for all $y$, if $|y|<|x|$, then $y \in S_{\pi(y)}$. Also we may assume that $\pi(x) \leqq|x|$; for otherwise $x \in S_{|x|+1} \subseteq S_{\pi(x)}$. By the same reasoning as in the preceding theorem, $\forall z P\left(x, z, S_{\alpha}\right)$ is true where $\alpha=\sup _{n \in \omega} \alpha_{n}$ and the $\alpha_{n}$ satisfy

$$
\alpha_{n} \geqq \sup _{z} \mu \beta\left[\beta \geqq \alpha_{n} \& P\left(x, z, S_{\beta}\right)\right] .
$$

We will make $\alpha<\pi(x)$. Suppose $\alpha_{n}$ is PC in $\boldsymbol{E},=, n, x$ (uniformly in $n$ ). We show that there exists an ordinal PC in $E,=, n, z, x$ (uniformly in $n, z$ ) bigger than $\mu \beta\left[\beta \geqq \alpha_{n} \& P\left(x, z, S_{\beta}\right)\right]$ by considering two cases.

Case 1. $|x|<\pi(x, z)$. This case is trivial since $|x| \geqq \alpha_{n} \& P\left(x, z, S_{|x|}\right)$.

Case 2. $\pi(x, z) \leqq|x|$. If the clause $b \in T$ occurs in $P(x, z, T)$, then $b \in S_{|x|} \Leftrightarrow$ $b \in S_{\pi(x, z)}$. For if $b \in S_{|x|}$, then $|b|<|x|$, so by the induction hypothesis $b \in S_{\pi(b)}$; but since $b$ is a term composed of $x, z$ and the pairing and projection functions, we have that $\pi(b) \leqq \pi(x, z)$; so $b \in S_{\pi(x, z)}$. The reverse implication follows from $\pi(x, z) \leqq|x|$. Thus since $P\left(x, z, S_{|x|}\right)$ is true, $P\left(x, z, S_{\pi(x, z)}\right)$ must be true and so (by the lemma) $P\left(x, z, S_{\beta}\right)$ is true for $\beta$ just below $\pi(x, z)$. Thus in either case there exist ordinals $\mathrm{PC}$ in $\boldsymbol{E},=, n, z, x$ (uniformly in $n, z$ ) bigger than $\mu \beta\left[\beta \geqq \alpha_{n} \&\right.$ $\left.P\left(x, z, S_{\beta}\right)\right]$. Using a selection operator, pick one; call it $\gamma_{n z}$. By the recursion theorem, construct $\alpha_{n+1} \geqq \sup _{z} \gamma_{n z}$. Since $\alpha=\sup _{n \in \omega} \alpha_{n}$ has ordinals above it PC in $E$, $=, x$, we see that $\alpha<\pi(x)$.

We close with the following remark. We have characterized semi-PC and semiSC rather than PC and SC. Of course, a PC [resp., SC] predicate is one that is semiPC [resp., semi-SC] and whose negation is as well. For the case of PC or SC in $E$, we can go a step further. Suppose $P(\mathfrak{a})$ is $P C$ in $E,=$. Then there is a set $S$ inductively defined with respect to a $\Pi_{1}$ predicate such that

$$
P(\mathfrak{a}) \Leftrightarrow\langle 0, \mathfrak{a}\rangle \in S, \quad \neg P(\mathfrak{a}) \Leftrightarrow\langle 1, \mathfrak{a}\rangle \in S .
$$

By using a selection operator, the smallest ordinals $\sigma_{\mathfrak{a}}$ such that $\langle 0, \mathfrak{a}\rangle \in S_{\sigma_{\mathfrak{a}}} \vee$ $\langle 1, \mathfrak{a}\rangle \in S_{\sigma_{\mathfrak{a}}}$ are PC in $\boldsymbol{E}$, $=, \mathfrak{a}$ (uniformly in $\mathfrak{a}$ ). Thus there is an ordinal $\sigma \geqq \sup _{\mathfrak{a}} \sigma_{\mathfrak{a}}$ that is $\mathrm{PC}$ in $E$, $=$ and $P(\mathfrak{a}) \Leftrightarrow\langle 0, \mathfrak{a}\rangle \in S_{\sigma}$. Therefore, the predicates $\mathrm{PC}$ in $E$, $=$ 
are precisely those reducible to a stage-whose ordinal is $\mathrm{PC}$ in $\boldsymbol{E},=$ - of a set inductively defined with respect to a $\Pi_{1}$ predicate. A similar remark holds for SC in $\boldsymbol{E},=$ by replacing $\Pi_{1}$ by $\Sigma_{2}$.

\section{REFERENCES}

1. K. J. Barwise, R. O. Gandy and Y. N. Moschovakis, The next admissible set, J. Symbolic Logic (to appear).

2. R. Fraïssé, Une notion de récursivité relative, Proc. Sympos. Infinitistic Methods, Foundations of Math. (Warsaw, 1959), Pergamon Press, Oxford; PWN, Warsaw, 1961, pp. 323-328. MR 32 \#3999.

3. C. E. Gordon, A comparison of abstract computability theories, J. Symbolic Logic 34 (1969), 156.

4. T. J. Grilliot, Selection functions for recursive functionals, Notre Dame J. Formal Logic 10 (1969), 225-234.

5. D. Lacombe, Deux généralisations de la notion de récursivité relative, C. R. Acad. Sci. Paris 258 (1964), 3410-3413. MR 28 \#3930.

6. R. Montague, Recursion theory as a branch of model theory, Logic, Methodology and Philosophy of Science III, North-Holland, Amsterdam, 1968, pp. 63-86.

7. Y. N. Moschovakis, Abstract first order computability. I, II, Trans. Amer. Math. Soc. 138 (1969), 427-504. MR 39 \#5362.

8. - Abstract computability and invariant definability, J. Symbolic Logic 34 (1969), 605-633.

9. - The Suslin-Kleene theorem for countable structures, Duke Math. J. 37 (1970), $341-352$.

10. Wayne Richter, Recursively Mahlo ordinals and inductive definitions (to appear).

11. C. Spector, Inductively defined sets of natural numbers, Proc. Sympos. Infinitistic Methods, Foundations of Math. (Warsaw, 1959), Pergamon Press, Oxford; PWN, Warsaw, 1961, pp. 97-102. MR 25 \#4991.

Pennsylvania State University, University Park, Pennsylvania 16802 